\title{
Effects of Random RF Disturbances on Aeronautical Communication Systems
}

\author{
E. Van Lil ${ }^{(1)}$, J. Verhaevert ${ }^{(2)}$, J. De Vos \& D. Van Troyen ${ }^{(3)}$ \\ (1) Div. ESAT-TELEMIC, KU Leuven, B-3001 Heverlee, Belgium \\ Emmanuel.VanLil@ESAT.KULeuven.Be \\ (2) Dept. Industrial Technology \& Construction, UGent, B-9000 Gent, Belgium \\ (3) Technologiecluster Elektrotechniek, KU Leuven, B-2860 Sint-Katelijne-Waver, Belgium
}

Air Traffic Control (ATC) and their responsible authorities have been always very sensitive to safety of the systems they are using to guarantee a fool-proof and environmentally safe operation of the facilities to provide air traffic services and guidance for the airplanes. This paper deals with the influence of random systems like badly shielded ignition systems of cars and motorbikes on standard air-ground aviation communication systems. Following a series of theoretical simulations, a measurement campaign quantifying this influence is described. This campaign is shedding some light on the fundamental behaviour and mechanisms of the RF disturbances caused by (shielded and unshielded) spark plug systems. It will be investigated both in the time domain and in the frequency domain. The frequency domain measurements were performed on an Open Area EMC Test Site a car of which a spark plug was removed so that the spark could be freely seen. The time domain measurements were performed both in situ and in a shielded EMC room. Also an analysis of the inaccuracies of those measurements and the working of the receiver is performed to explain the real effect of those disturbances on practical communication systems from aircraft to ground receivers with high $(20 \mathrm{~m})$ towers with vertically polarised antennas. We were able to prove experimentally that the effect of a signal, due to the A(utomatic)G(ain)C(ontrol) of the receiver, was, due to an RC filter integration, proportional to the product of the field strength and the duty cycle. So, a signal with a duty cycle of $5 \%$ must be 20 times larger in amplitude or 400 times larger in power than an equivalent $\mathrm{CW}$ signal before it has a significant effect on the receiver (a small background noise equal to the $\mathrm{P}($ ulse)R(epetition)F(requency) of the pulsed signal may be audible, but usually it is less than the cockpit background noise in the airplane).

\section{ACKNOWLEDGMENT}

We are greatly indebted to the directors and personnel of Belgocontrol, who funded this research and gave us all information on their communication systems. 